\title{
The Role of Angiopoietine-2 in the Diagnosis and Prognosis of Sepsis
}

\author{
Janos Szederjesi¹, Emoke Almasy¹, Alexandra Lazar ${ }^{1}$, Adina Huțanu², Anca Georgescu3* \\ 1 University of Medicine and Pharmacy Tîrgu Mureş, Romania, Discipline of Intensive Care, 38 Gheorghe Marinescu \\ street, Tirgu Mures, 540139, Romania \\ ${ }^{2}$ County Clinical Emergency Hospital Tirgu Mures, Romania, 50 Gheorghe Marinescu street Tirgu Mures, 540139, \\ Romania \\ 3 University of Medicine and Pharmacy Tîrgu Mureş, Romania, Discipline of Infectious Diseases, 38 Gheorghe \\ Marinescu street, Tirgu Mures, 540139, Romania
}

\begin{abstract}
Introduction: Angiopoietin-2 (ANG-2) is a new biomarker whose blood-serum values increase in sepsis and its expression is elevated in line with the severity of the degree of inflammation. The aim of this study was to identify the diagnostic role of ANG-2 in patients with non-surgical sepsis addmitted to an intensive care unit.

Material and methods: This was a prospective randomized study including 74 patients admitted in the Clinic of Intensive Care of the County Clinical Emergency Hospital Tirgu Mureș, divided into two groups: Group S: patients with sepsis ( $n=40,54 \%$ ) and Group C: control, without sepsis ( $n=34,46 \%)$. ANG-2 levels were determined in both groups.

Results: From the Group S, 14 patients (35\%) had positive haemocultures. ANG-2 values varied between 1 and 43 $\mathrm{ng} / \mathrm{mL}$, with an average of $6.0 \mathrm{ng} / \mathrm{mL}$ in patients without sepsis and $10.38 \mathrm{ng} / \mathrm{mL}$ in patients with sepsis $(\mathrm{p}=0.021)$. A positive correlation between ANG-2 and SAPS II, SOFA and APACHE II severity scores was demonstrated, as was a positive correlation between serum levels of ANG-2 and procalcitonine. ANG-2 had a $5.71 \%$ specificity and $74.36 \%$ sensitivity for diagnosis of sepsis.
\end{abstract}

Conclusions: ANG-2 serum levels were elevated in sepsis, being well correlated with PCT values and prognostic scores. ANG-2 should be considered as a useful biomarker for the diagnosis and the prognosis of this pathology.

Keywords: Angiopoietin-2, sepsis, infection

Received: 04 January 2015 / Accepted: 15 January 2015

\section{INTRODUCTION}

Sepsis is a pathology frequent encountered in intensive care units, occuring as a major complication in the development of serious infections. Severe sepsis, defined as a sepsis associated with organ dysfunction [1] is associated with a high mortality rate [2] and is caused by an infection induced immune response [3]. Diagnosis is based on clinical criteria, the principle indicator being a systemic inflammatory response syndrome (SIRS), triggered by an infection [4,5]. The prognosis for these patients is dependant on the early establishment of the proper diagnosis and the quick initiation of antibiotic therapy [6]. Recently, special attention has been given to new biomarkers associated with sepsis. Some of these have proved to be effective in in the di- agnosis and prognosis of this severe disease. Angiopoietin-2 (ANG-2) is one of these biomarkers, and has been intensively studied. It is produced by endothelial cells from Weibel-Palade corpusclues. ANG-2 induces inflammation of endothelial cells, as well as vessel regression and distruction. The blood-serum values of ANG-2 increase in sepsis and its expression is elevated in line with the severity of the degree of inflammation. However high expression of this biomarker is also found in other pathologies such as multiple mieloma [7], squamos cells carcinoma [8] or heart failure in patients on dialysis [9]. The mechanism of action of Angiopoietin-2 involves the tyrosine kinase receptor (TIE2) [10].

The aim of this study is to identify the diagnostic role of ANG-2 in patients with non-surgical sepsis ad-

\footnotetext{
* Correspondence to: Anca Georgescu, University of Medicine and Pharmacy Tîrgu Mureș, Romania, Discipline of Infectious Diseases, 38 Gheorghe Marinescu street Tirgu Mures, 540139, Romania; e-mail: ancameda.georgescu@umftgm.ro
} 
mitted to an intensive care unit. We tested the hypothesis that ANG-2 has a high sensitivity and specificity for the diagnosis of sepsis and for the prognosis of this disease.

Objectives of the study were:

- To asses the prognostic role of ANG-2 in sepsis.

- To study the correlation between ANG-2 and procalcitonine (PCT).

- To study the correlation between ANG-2 and the following score systems:

a. APACHE II score (Acute Physiology and Chronic Health Evaluation),

b. SOFA (Sequential Organ Failure Assessment)

c. SAPS II (Simplified Acute PhysiologyScore).

- To study the correlation between ANG-2 and the requirements for vasoactive medication.

- To study the correlation between ANG-2 and renal function.

\section{MATERIAL AND METHODS}

This is a prospective randomized study including 74 patients admitted in the Clinic of Intensive Care of the County Clinical Emergency Hospital Tirgu Mureș in the period of January to November 2014. The study was approved by the Ethics Commitee of the University of Medicine and Pharmacy of Tirgu Mures and all the patients or their relatives were given a clear explanation of the study and gave their informed consent before randomization.

Inclusion criteria: All patient, of either sex, admitted to the intensive care ward, who were over the age of 18 years and had no associated pathology or had received any surgical intervention in the previous 72 hours, were admitted into the study.

Exclusion criteria: patients with cardiac arrest or associated pathologies that could cause expression of high levels of ANG-2.

On admission to the intensive care unit a medical history was taken and the following tests carried out:

a. record of status of vital signs

b. laboratory tests including cell blood count, creatinine, urea, coagulation parameters and liver transaminase

A clinical diagnosis was made at this point. Blood cultures and bacteriological determinations were used to confirm the diagnosis.
Blood samples were collected directly via vascular puncture after skin desinfection with betadine. The blood cultures were accomplished using separate vials for aerob germs (Standard SA) and anaerobs (Standard $\mathrm{SN})$. Analyses of the blood cultures were processed using the BacT/Alert 3D (Biomerieux, France) automated haemoculture system.

APACHE II, SOFA and SAPS II severity scores were calculated and serum levels of PCT, C-Reactive protein (CRP) and ANG-2 were determined in the first 12 hours after admission.

The blood samples were frozen at $-70^{\circ} \mathrm{C}$ and processed later.

ANG-2 expression was evaluated using the EnzymeLinked Immuno Sorbent Assay (ELISA) test (R\&D Systems, Minneapolis, USA).

PCT, CRP, the sepsis-related organ failure assessment was determined using the immunoturbidimetry method (Cobas 6000, Roche Diagnostics, Germany) and PromoKinekits for the detection, elimination and prevention of cell culture contamination (PromoCell $\mathrm{GmbH}$, Heidelberg, Germany).

Patients were divided into two groups: Group S: patients with sepsis $(n=40,54 \%)$ and Group C: control, without sepsis ( $n=34,46 \%)$.

A diagnosis of sepsis to be accepted, all three of the following criterion had to be present:

a. Two clinical criteria for SIRS

b. PCT higher than $0.2 \mathrm{ng} / \mathrm{mL}$

c. confirmed infection.

Statistical analysis was performed using Microsoft Excel (Microsoft, Washington, USA), GraphPad (GraphPad Software, Inc., California, USA) and MedCalc (MedCalc Software, Ostend, Belgium).

Analysis included specificity and sensibility of ANG-2 for diagnosis of sepsis, and correlation with the above mentioned parameters and death. Graphic representation of receiver-operating-characteristic (ROC) were used, with determination of area under the curve (AUC). Quantitative variables were tested for normal distribution using the Kolmogorov-Smirnov test and Bartlets Test for equal variances. Where applicable the Mann-Whitney or Pearson tests were used for variable correlations. Pearson's chi ${ }^{2}$ test with Fisher or Yates correction were used for comparing distribution of nominal values. A p value $<0.05$ was considered statistically significant. 


\section{RESULTS}

Seventy four patients were enrolled in the study, of which 40 patients (54\%) fulfilled the criteria for sepsis, and 34 (46\%) were included in the control group. From the Group S, 14 patients (35\%) had positive haemocultures. ANG-2 values varied between 1 and $43 \mathrm{ng} / \mathrm{mL}$, with an average of $6.0 \mathrm{ng} / \mathrm{mL}$ in patients without sepsis and $10.38 \mathrm{ng} / \mathrm{mL}$ in patients with sepsis $(\mathrm{p}=0.021)$ (Figure 1).

Demographic and paraclinical data of the patients in the two groups are presented in Table 1.

A positive correlation between ANG-2 and SAPS II, SOFA and APACHE II severity scores was demonstrated, as was a positive correlation between serum levels of ANG-2 and PCT (Table 2).

The lungs were the most frequent locus of infection in the study groups (Table 3 ).

ANG-2 was both "specific" and "sensitive” in the diagnosis of sepsis: $65.71 \%$ specificity and $74.36 \%$ sensitivity (Figure 2).

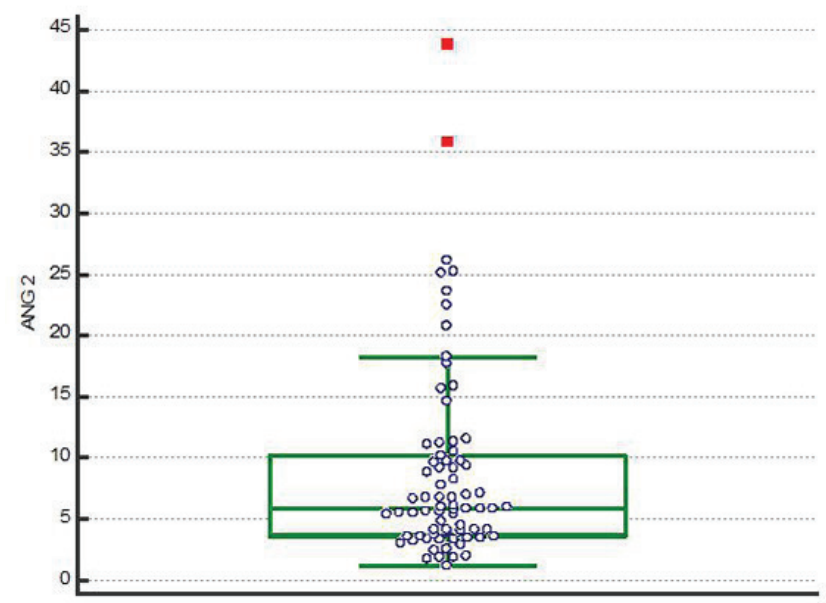

Figure 1. ANG-2 values in the studied patients

The cut-off value ANG-2 for diagnosis of sepsis was established at $5.61 \mathrm{ng} / \mathrm{mL}$ according to the Youden test.

A high mortality rate of $75.67 \%(n=56)$ was recorded in those cases where ANG-2 showed a high specificity $(88.89 \%)$ together with a lower sensitivity $(35.71 \%)$ $(\mathrm{p}=0.195)$ (Figure 3).

Table 1. Demographic and paraclinical data in the two groups of patients

\begin{tabular}{|c|c|c|c|}
\hline Parameter & $\begin{array}{c}\text { Group -S } \\
\text { (Patients with sepsis) } \\
\text { (n=40, 54\%) }\end{array}$ & $\begin{array}{c}\text { Group- C } \\
\text { (Control. Patients } \\
\text { without sepsis) } \\
(n=34,46 \%)\end{array}$ & P value \\
\hline Age (years, average \pm SD) & $76 \pm 15.87$ & $68 \pm 13.39$ & 0.0525 \\
\hline Gender, male (\%) & $24(60 \%)$ & $12(35.29 \%)$ & 0.0393 \\
\hline BMI $\left(\mathrm{kg} / \mathrm{m}^{2}\right.$, average $\left.\pm \mathrm{SD}\right)$ & $24.6 \pm 8.34$ & $26.9 \pm 8.77$ & 0.1300 \\
\hline Days in intensive care unit TI (average \pm SD) & $2 \pm 4.6$ & $10 \pm 10.33$ & $<0.0001$ \\
\hline Days under vasoactive treatment (average \pm SD) & $1 \pm 1.73$ & $3 \pm 3.35$ & 0.0073 \\
\hline Patients requiring vasoactive treatment $(n, \%)$ & $33(82.5 \%)$ & $28(82.23 \%)$ & 0.434 \\
\hline Mortality (n, \%) & $32(80 \%)$ & $24(70.59 \%)$ & 0.4197 \\
\hline ANG-2 (ng/mL, average \pm SD) & $7.37 \pm 9.21$ & $4.11 \pm 4.34$ & 0,0005 \\
\hline $\mathrm{PCT}(\mathrm{ng} / \mathrm{mL}$, average $\pm \mathrm{SD})$ & $1.525 \pm 3.03$ & $0.71 \pm 0.75$ & $<0.0001$ \\
\hline $\mathrm{CRP}(\mathrm{ng} / \mathrm{mL}$, average $\pm \mathrm{SD})$ & $164.4 \pm 126.7$ & $82.71 \pm 78.98$ & $<0.0001$ \\
\hline Haemoglobin (g/dL, average \pm SD) & $12.35 \pm 3.09$ & $11.75 \pm 1.84$ & 0.2762 \\
\hline Haematocrit (\%, average \pm SD) & $33.2 \pm 8.99$ & $34.05 \pm 9.84$ & 0.6879 \\
\hline Leucocytes (x1000/mm³, average \pm SD) & $16.35 \pm 12.44$ & $13.95 \pm 9.7$ & 0.6027 \\
\hline Creatinine (mg/dL, average \pm SD) & $1.84 \pm 3.37$ & $0.82 \pm 1.88$ & 0.0005 \\
\hline Ureea (mg/dL, average \pm SD) & $104.9 \pm 98.21$ & $62.95 \pm 58.68$ & 0.0168 \\
\hline APACHE II (val, average \pm SD) & $27 \pm 8.8$ & $23 \pm 7.74$ & 0.0152 \\
\hline SOFA (val, average \pm SD) & $9 \pm 3.65$ & $7 \pm 3.07$ & 0.0616 \\
\hline SAPS (val, average \pm SD) & $51.5 \pm 20.18$ & $42 \pm 14.77$ & 0.0276 \\
\hline
\end{tabular}

\footnotetext{
* $p$ value calculated using Mann-Whitney test (significant for $p<0,05$ ).
} 
Table 2. Correlations between ANG-2and PCT levels, severity scores, days of vasoactive medication and renal function

\begin{tabular}{lcl} 
Parameter & Correlation coefficient $*^{*}$ & P value \\
\hline PCT & -0.3049 & 0.0223 \\
\hline APACHE II & 0.2342 & 0.0446 \\
\hline SOFA & 0.2603 & 0.0251 \\
\hline SAPS & 0.3321 & 0.0038 \\
\hline Patients with vasoactive medication & 0.2353 & 0.0436 \\
\hline Days of vasoactive medication & -0.08736 & 0.4656 \\
\hline Creatinine & -0.256811 & 0.0560 \\
\hline Ureea & -0.2200918 & 0.1031
\end{tabular}

* Spearman correlation coefficient on the entire lot of patients $(n=74)$.

Table 3. Site of infection in the studied groups

\begin{tabular}{lcc} 
Infection localisation & Sepsis group (n=40) & No sepsis group (n=34) \\
No infection & 0 & $10(29.41 \%)$ \\
\hline Pulmonar & $26(65 \%)$ & $15(44.11 \%)$ \\
\hline Abdominal & $4(10 \%)$ & $4(11.76 \%)$ \\
\hline Urinar tractus & $2(5 \%)$ & $2(5.88 \%)$ \\
\hline Articular & 0 & $1(2.94 \%)$ \\
\hline Surgical wound & 0 & $1(2.94 \%)$ \\
\hline Central Nervous System & 0 & $1(2.94 \%)$ \\
\hline Cutanat & $6(15 \%)$ & 0 \\
\hline Undefined & $2(5 \%)$ & 0
\end{tabular}

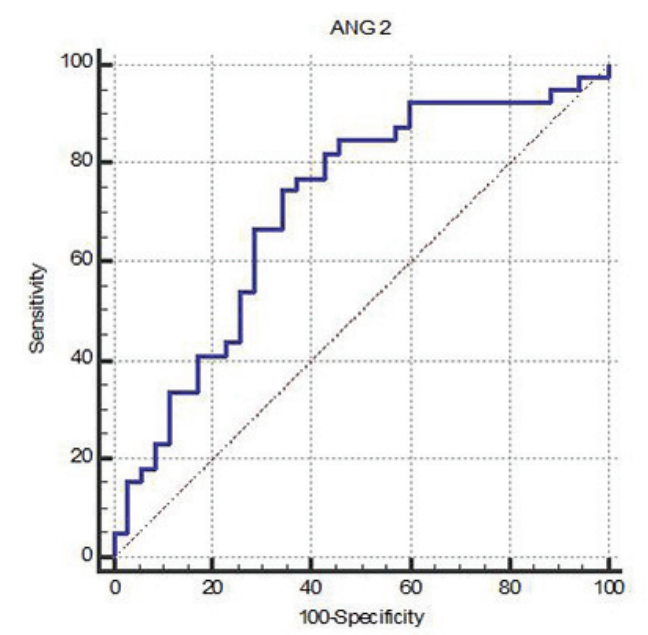

\begin{tabular}{lc}
\hline Area under the ROC curve (AUC) & 0.710 \\
\hline Standard Error & 0.0620 \\
\hline Youden index & 0.4007 \\
\hline Association criteria & $>5.6162$ \\
\hline $95 \%$ Confidence interval & 0.593 to 0.810 \\
\hline Significance level P (Area=0.5) & 0.0007 \\
\hline Sensitivity & 74.36 \\
\hline Specificity & 65.71 \\
\hline
\end{tabular}

Figure 2. ROC curve for specificity and sensitivity of ANG 2 for diagnosis of sepsis

There was no statistically significant correlation between ANG-2 levels and the number of days on vasoactive medication ( $\mathrm{p}=0.465)$. However, ANG-2 serum levels were correlated with the number of patients who required vasoactive treament $(\mathrm{p}=0.043)$. A statistically significant correlation was found between ANG-2 levels and serum levels of both creatinine and urea. 


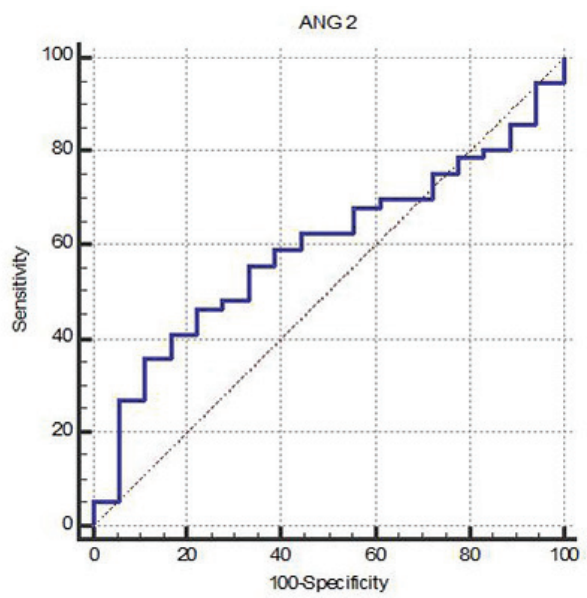

\begin{tabular}{lc}
\hline Area under the ROC curve (AUC) & 0.591 \\
\hline Standard Error & 0.0705 \\
\hline 95\% Confidence interval & 0.471 to 0.704 \\
\hline Significance level P (Area=0.5) & 0.1957 \\
\hline Sensitivity & 35.71 \\
\hline Specificity & 88.89
\end{tabular}

Figure 3. ROC curve for specificity and sensitivity of ANG 2 for predicting mortality rate

\section{DISCUSIONS}

\section{ANG-2 and diagnosis of sepsis}

This study demonstrated that ANG-2 has a high sensitivity and specificity for the diagnosis of sepsis and for the prognosis of sepsis.

ANG-2 levels obtained in this study were higher than those described by other authors. Buddingh et al [11] reported an average value of $3 \mathrm{ng} / \mathrm{mL}$ ANG-2 in healthy subjects, which increased to double values in patients with severe acute pancreatitis and severe organ dysfunction, concluding that this value was highly predictive for disease severity. In the current study, the values of ANG-2 in the control group were higher $(6.0 \mathrm{ng} /$ $\mathrm{mL})$, probably due to the fact that patients with infections, but without sepsis, were included in this group. Nevertheless, the statistically significant difference in ANG-2, between the two groups, proves that this biomarker can be useful for assessment of severity of infection in agreement with other stufies [12-14].

In the current study, a cut-off value of $5.61 \mathrm{ng} / \mathrm{mL}$ was the borderline value below which the probability of infection was very low, this being similar to previously reported value of $6.0 \mathrm{ng} / \mathrm{mL}$, reported by Buddingh et al [11] reported as representing the cutoff point above which being indicative of patients with an infection.

Several authors reported a good correlation between ANG-2 levels and pulmonary function in patients treated with high doses of interleukine-2 [15].

\section{ANG-2, duration of stay in ICU and the requirements for vasoactive medication}

Analysis of the results of the present study showed that patients without sepsis were retained intensive care units longer that those with sepsis. This apparent anomaly may be explained by the fact that sepsis patients presented a higher mortality rate, death occuring frequently in the first days after admission. A similar trend was noticed in regards to the number of days patients were maintained on vasoactive treatment.

An increase in cardiac output, related to a descrease in the left ventricular ejection fraction and hypotension has been associated with an increase of serum levels of ANG-2 [16].

\section{ANG-2 levels and renal function.}

The present study was unable to demonstrate a clinically significant correlation between ANG-2 and renal function in contradiction to the report by Tsai et al which showed ANG to be an appropriate predictor of the unfavourable development of renal failure eventhough in the current stage the exact pathophysiological mechanism for this effect has not been clearly identified [17].

\section{ANG-2 levels and severity scores}

Another objective of the study was to demonstrate the correlation between ANG-2 and the severity scores. We proved that ANG-2 values correlate very well with the APACHE II, SOFA and SAPS severity scores, used in the study. This supports the role ANG-2 as a biomarker for prognosis of severe sepsis, all be it that the sensitivity of this parameter for prediction of death is not high (35\%). Again, this low value may reflect the fact that patients without sepsis have a high mortality rate $(70.59 \%)$ due to other pathologies. 
Available online at: www.jccm.ro

AGT-2 differentially regulates angiogenesis through tyrosine kinase-2 receptors (TIE2) and integrin signallingand several studies have demonstrated that not only the ANG-2 levels, but also TIE2 levels, may be usefull in determining the prognosis of patients with severe sepsis, as the vascular modelling determined by ANG-2 is well correlated with the levels of TIE2 [18].

\section{- CONCLUSIONS}

ANG-2 serum levels were elevated in sepsis, being well correlated with PCT values and prognostic scores. ANG-2 is both high specific and sensitive for the diagnosis of sepsis, and should be considered as a useful biomarker for the diagnosis and the prognosis of this pathology.

\section{ACKNOWLEDGEMENTS}

This paper is supported by the Sectoral Operational Programme Human Resources Development (SOP HRD), financed from the European Social Fund and by the Romanian Government under the contract number POSDRU/159/1.5/S/133377.

\section{CONFLICT OF INTEREST}

The authors declare that they have no conflict of interest.

\section{- REFERENCES}

1. Surviving Sepsis Campaign Guidelines Committee including The Pediatric Subgroup; Dellinger, RP; Levy, MM; Rhodes, A et al. Surviving Sepsis Campaign: International guidelines for management of severe sepsisand septic shock: 2012. Critical Care Medicine. 2013;41:580-637.

2. Russel, JA. The current management of septic shock. Minerva Medica. 2008;99:431-58.

3. Deutschman, CS; Tracey, KJ. Sepsis: Current dogma andnewperspectives. Immunity. 2014;40:463-75.

4. Jui J. Septic Shock. In Tintinalli JE, Stapczynski JS, Ma OJ et al. Tintinalli's Emergency Medicine: A Comprehensive Study Guide (7th ed.). New York: McGraw-Hill. 2011;pp.1003-14.

5. Dellinger RH, Levy MM, Rhodes $A$, et al. Surviving Sepsis
The Journal of Critical Care Medicine 2015;1(1) • 23

Campaign: International guidelines for management of severe sepsisand septic shock: 2012. Crit Care Med. 2013;41:580-637.

6. Bone R, Balk R, Cerra F, et al. Definitions for sepsisand organ failure and guidelines for the use of innovative therapies in sepsis. The ACCP/SCCM Consensus Conference Committee. American College of Chest Physicians/Society of Critical Care Medicine. Chest. 1992;101:1644-55.

7. Pappa CA, Tsirakis G, Samiotakis P, et al. Serum levels of angiopoietin-2 are associated with the growth of multiple myeloma. Cancer Invest. 2013;31:385-9.

8. Li C, Sun CJ, Fan JC, et al. "Angiopoietin-2 expression is correlated with angiogenesis and overall survival in oral squamous cell carcinoma. Med Oncol. 2013;30:571.

9. Shroff RC, Price KL, Kolatsi-Joannou M, et al. Circulating angiopoietin-2 is a marker for early cardiovascular disease in children on chronic dialysis. PLoS ONE. 2013;8:e56273.

10.Jeansson M, Gawlik A, Anderson G, et al. Angiopoietin-1 is essential in mouse vasculature during development and in response to injury. J Clin Invest. 2011;121:2278-89.

11. Buddingh KT, Koudstaal LG, van Santvoort HC, et al. Early angiopoietin-2 levels after onset predict the advent of severe pancreatitis, multiple organ failure, and infectious complications in patients with acute pancreatitis. J Am Coll Surg. 2014;218:26-32.

12.Wang K, Bhandari V, Giuliano JS Jr, O Hern CS, Shattuck MD, Kirby M. Angiopoietin-1, angiopoietin-2 and bicarbonate as diagnostic biomarkers in children with severe sepsis. PLoS One. 2014;25:e108461.

13. Mearelli F, Fiotti N, Altamura N, et al. Heterogeneous models for an early discrimination between sepsis and non-infective SIRS in medical ward patients: a pilot study. Intern Emerg Med. 2014;9:749-57.

14.Giuliano JS Jr, Tran K, Li FY, Shabanova V, Tala JA, Bhandari V. The temporal kinetics of circulating angiopoietin levels in children with sepsis. Pediatr Crit Care Med. 2014;15:e1-8.

15.Gores KM, Delsing AS, Kraus SJ, et al.Plasma Angiopoietin-2 concentrations are related to impaired lung function and organ failure in a clinical cohort receiving high-dose interleukin 2 therapy. Shock. 2014;42:115-20.

16.Ziegler T, Horstkotte J, Schwab C, et al. Angiopoietin-2 mediates microvascular and hemodynamic alterations in sepsis. J Clin Invest. 2013. pii: 66549.

17.Tsai YC, Chiu YW, Tsai JC, et al. Association of angiopoietin-2 with renal outcome in chronic kidney disease. PLoS One. 2014;3;9:e108862.

18. Felt $M$, Luck $R$, Schering $A$, et al. Angiopoietin-2 differentially regulates angiogenesis through TIE2 and integrin signaling. J Clin Invest. 2012;122:1991-2005. 